\title{
Cytotoxic Effects of Carbon Nanotubes and Cisplatin Conjugates on 3D Breast Cancer Cellular Models ${ }^{\dagger}$
}

\author{
Mădălina Andreea Badea 1,*, Mihaela Balas 1, Daniela Ioniță ${ }^{2}$ and Anca Dinischiotu 1 \\ 1 Department of Biochemistry and Molecular Biology, Faculty of Biology, University of Bucharest, \\ 91-95 Splaiul Independentei, R-050095 Bucharest, Romania; mihaela.radu@bio.unibuc.ro (M.B.); \\ anca.dinischiotu@bio.unibuc.ro (A.D.) \\ 2 Department of General Chemistry, Faculty of Applied Chemistry and Materials Science, Politehnica \\ University of Bucharest, 313 Splaiul Independentei, 060042 Bucharest, Romania; \\ daniela.ionita@upb.ro (D.I.) \\ * Correspondence: madalina.andreea.badea@drd.unibuc.ro \\ + Presented at the 15th International Symposium "Priorities of Chemistry for a Sustainable Development" \\ PRIOCHEM, Bucharest, Romania, 30th October-1st November 2019.
}

Published: 9 October 2019

Keywords: carbon nanotubes; MCTSs; cisplatin; cytotoxicity

Breast cancer represents one of the leading causes of death for which new strategies of treatment are intensively studied and tested. Single-walled carbon nanotubes (SWCNTs) represent promising tools in the treatment of cancer and can be used as transporters of drugs due to their unique properties [1]. Furthermore, advanced cellular models, such as multicellular tumor spheroids (MCTSs), are exploited for the study of cancer mechanisms. MCTSs represent 3D cellular structures composed by three layers of cells found in different stages of the cell cycle: the first one is characterized by proliferative cells, followed by cells in a quiescent state, and the last one in the spheroid's center is represented by necrotic cells [2]. In this context, we aimed to investigate the cytotoxic effects of SWCNTs loaded with cisplatin (CDDP) in breast cancer MCTSs.

SWCNTs were functionalized with carboxyl groups, resulting in SWCNT-COOH, which was mixed with dimethylformamide and CDDP to obtain the SWCNT-COOH-CDDP nanoconjugates. SWCNT-COOH-CDDP was characterized by inductively coupled plasma mass spectrometry, Fourier-transform infrared spectroscopy (FTIR), energy-dispersive X-ray spectroscopy (EDX), and Raman spectroscopy. MCTSs were generated from MDA-MB-231 breast cancer cells in Dulbecco's Modified Eagle Medium with 2.5\% Matrigel, using Nunclon ${ }^{\mathrm{TM}}$ Sphera ${ }^{\mathrm{TM}}$ Microplates. On the second day of culture, MCTSs were treated with doses of $1,4 \mu \mathrm{g} / \mathrm{mL}$ SWCNTs and 0.6, $2.52 \mu \mathrm{g} / \mathrm{mL}$ CDDP for 24 and $48 \mathrm{~h}$. Untreated MCTSs were used as the control. The MCTSs' morphology was analyzed using optical microscopy while cellular viability was assessed through a fluorescence method, using LIVE/DEAD assay. Cell death was evaluated by analyzing the expression of pro-apoptotic (Bax) and autophagic (Beclin-1) proteins using the Western blot technique.

Our results indicated that the concentration of CDDP encapsulated in SWCNT-COOH is 192.82 $\mu \mathrm{g} / \mathrm{mL}$. Also, the EDX spectrum highlighted the presence of Pt ions in the SWCNT-COOH-CDDP sample while the FTIR results indicated a covalent bond between CDDP and SWCNT-COOH. The analysis of optical microscopy images indicated a reduction in the size of SWCNT-COOH-CDDP-treated MCTSs and the detachment of cells from the proliferative layer after $48 \mathrm{~h}$, compared with the effects induced by free components, which presented high biocompatibility. Fluorescence images revealed the presence of a necrotic center surrounded by live cells in all tested conditions. However, when MCTSs were treated with $4 \mu \mathrm{g} / \mathrm{mL}$ SWCNT-COOH-CDDP, the detachment of cells from the edge was noticed, in correlation with 
optical microscopy images. The expression of the Bax protein increased after 24 and $48 \mathrm{~h}$ of exposure to $4 \mu \mathrm{g} / \mathrm{mL}$ SWCNT-COOH-CDDP relative to the control but not in the presence of 1 $\mu \mathrm{g} / \mathrm{mL}$ SWCNT-COOH-CDDP. In the same time, the expression of Beclin-1 was inhibited after 24 and $48 \mathrm{~h}$ of exposure with $1 \mu \mathrm{g} / \mathrm{mL}$ and $4 \mu \mathrm{g} / \mathrm{mL}$ SWCNT-COOH-CDDP, respectively. A slight increase of Beclin-1 expression was observed after the treatment of MCTSs with $4 \mu \mathrm{g} / \mathrm{mL}$ SWCNT-COOH-CDDP for $48 \mathrm{~h}$. No significant changes were observed in the presence of $1 \mu \mathrm{g} / \mathrm{mL}$ SWCNT-COOH-CDDP after $48 \mathrm{~h}$.

Considering the results of the present study, we conclude that SWCNT-COOH-CDDP nanoconjugates have the potential to induce significant morphological changes in MCTSs and to initiate apoptosis pathway through the activation of the Bax protein and the inhibition of the autophagy process.

Acknowledgments: This work was supported by a grant of Ministry of Research and Innovation, CNCSUEFISCDI, project number PN-III-P2-2.1-PED-2016-0904, within PNCDI III.

\section{References}

1. Rastogi, V.; Yadav, P.; Bhattacharya, S.S.; Mishra, A.K.; Verma, A.; Pandit, J.K. Carbon nanotubes: An emerging drug carrier for targeting cancer cells. J. Drug Deliv. 2014, 670815, doi:10.1155/2014/670815.

2. Cui, X.; Hartanto, Y.; Zhang, H. Advances in Multicellular Spheroids Formation. J. R. Soc. Interface 2017, 20160877, doi:10.1098/rsif.2016.0877.

(C) 2019 by the authors. Licensee MDPI, Basel, Switzerland. This article is an open access article distributed under the terms and conditions of the Creative Commons Attribution (CC BY) license (http://creativecommons.org/licenses/by/4.0/). 